\title{
Scanning tunneling microscopy and spectroscopy of semi-insulating GaAs
}

\author{
N. D. Jäger, ${ }^{1}$ Ph. Ebert, ${ }^{1}$ K. Urban, ${ }^{1}$ R. Krause-Rehberg, ${ }^{2}$ and E. R. Weber ${ }^{3}$ \\ ${ }^{1}$ Institut für Festkörperforschung, Forschungszentrum Jülich GmbH, 52425 Jülich, Germany \\ ${ }^{2}$ Fachbereich Physik, Martin-Luther-Universität Halle, 06099 Halle, Germany \\ ${ }^{3}$ Department of Materials Science, University of California, and Materials Science Division, Lawrence Berkeley National Laboratory, \\ Berkeley, California 94720
}

(Received 3 January 2002; published 10 May 2002)

\begin{abstract}
We report on atomically resolved scanning tunneling microscopy images and tunneling spectra of (110) cleavage surfaces of semi-insulating GaAs without illumination at room temperature. With help of simple model calculations we extract the physical mechanisms involved in the tunneling processes from and into semi-insulating GaAs. Atomically resolved images can only be observed at negative voltages, while no tunneling into empty states is possible without illumination. This is explained, on the one hand, by the absence of a carrier inversion at the semiconductor surface without illumination under the nonequilibrium tunneling contact conditions. On the other hand, at negative voltages in the noncontact mode an accumulation at the surface occurs and leads to tunneling of electron from the valence band states into the empty tip states. This current is limited by the tunneling through the vacuum barrier and the scanning tunneling microscopy images are found to show the occupied dangling bond states above the arsenic atoms. In the point contact mode the current is limited by tunneling through the space charge region without and with illumination. The implications of the results for the investigation of low-conductivity materials by scanning tunneling microscopy are discussed.
\end{abstract}

DOI: 10.1103/PhysRevB.65.195318

PACS number(s): 73.20.At, 85.60.- q

\section{INTRODUCTION}

Semiconductor heterostructures and devices are frequently grown on or implanted in semi-insulating substrates or may contain semi-insulating layers in order to electrically decouple different active areas on the same chip. For a further optimization of such devices, it is desirable to achieve an atomically resolved analysis of the entire device structure including the semi-insulating layers or components. One of the most successful techniques for such an atomistic characterization is cross-sectional scanning tunneling microscopy (XSTM), which provided excellent geometric and electronic data of semiconductor heterostructures. ${ }^{1-8}$ Scanning tunneling microscopy can, however, only be applied on electrically conducting materials. So far bulk "materials with insufficient conductivity at room temperature" ${ }^{9}$ could only be imaged with additional carrier generation at elevated temperatures or by light illumination. ${ }^{10}$

In this paper we demonstrate that cleavage surfaces of semi-insulating GaAs can be imaged with atomic resolution by STM without light illumination or additional heating. We determine the origin of the current and discuss the physical effects involved. This possibility of directly imaging even semi-insulating GaAs allows to investigate all types of semiconductor heterostructures including those with semiinsulating substrates or layers by XSTM.

\section{EXPERIMENT}

For our experiment we used two different types of semiinsulating GaAs wafers supplied by Freiberger Compound Materials and American Crystal Technology (AXT). The Freiberger Compound Materials wafer contained carbon and compensating EL2 centers in concentrations of $4.8 \times 10^{14}$ and $1.3 \times 10^{16} \mathrm{~cm}^{-3}$, respectively. The EL2 concentration is sufficient to compensate all shallow donors and acceptors. The supplier specifies a resistivity $\rho$ of $1.2 \times 10^{7} \Omega \mathrm{cm}$ measured by the Hall-van der Pauw method at $22^{\circ} \mathrm{C}$ with a $n$-type conduction. The AXT wafer had a specification as semi-insulating ( $\rho>10^{7} \Omega \mathrm{cm}$ ). One side of the wafers had an ohmic contact consisting of $\mathrm{Ni} / \mathrm{AuGe} / \mathrm{Ni} .{ }^{11}$ Bars $(3 \mathrm{~mm}$ wide and $8 \mathrm{~mm}$ high) were cleaved out of the $380-\mu \mathrm{m}$-thick wafer. The lower half of the bars was clamped entirely between gold contacts, transferred into ultrahigh vacuum (pressure $<1 \times 10^{-8} \mathrm{~Pa}$ ), and cleaved perpendicular to a [110] direction at a scratch mark just above the clamp. Within $2-10 \mathrm{~h}$ after the cleavage the surfaces were investigated by a home-made "beetle type" STM using a RHK STM-100 electronics. The preamplifier used had a combined noise and offset level of about $30 \mathrm{pA}$. PtRh tips sharpened by an electrochemical molten salt etch were used. All STM images were obtained at a constant tunneling current of $1 \mathrm{nA}$ with a scanning speed of $100-300 \mathrm{~nm} / \mathrm{s}$, in the dark, and at room temperature. Current-voltage tunneling spectra were acquired at constant tip height with a set point of $1 \mathrm{nA}$. The entire voltage range was swept in $300-340 \mathrm{~ms}$ and the current was recorded in $15 \mathrm{mV}$ intervals. Each curve presented arises from a single sweep.

\section{EXPERIMENTAL RESULTS}

Figure 1 shows several constant-current scanning tunneling microscope images acquired at different magnitudes of negative tunneling voltages applied to the sample. The images all exhibit a periodic pattern of maxima with unit-cell dimensions of 0.56 and $0.4 \mathrm{~nm}$ along the [001] and [1 $1 \overline{0}$ ] directions, respectively, independent of the tunneling voltage. The size of the unit cell and the morphology of the STM 

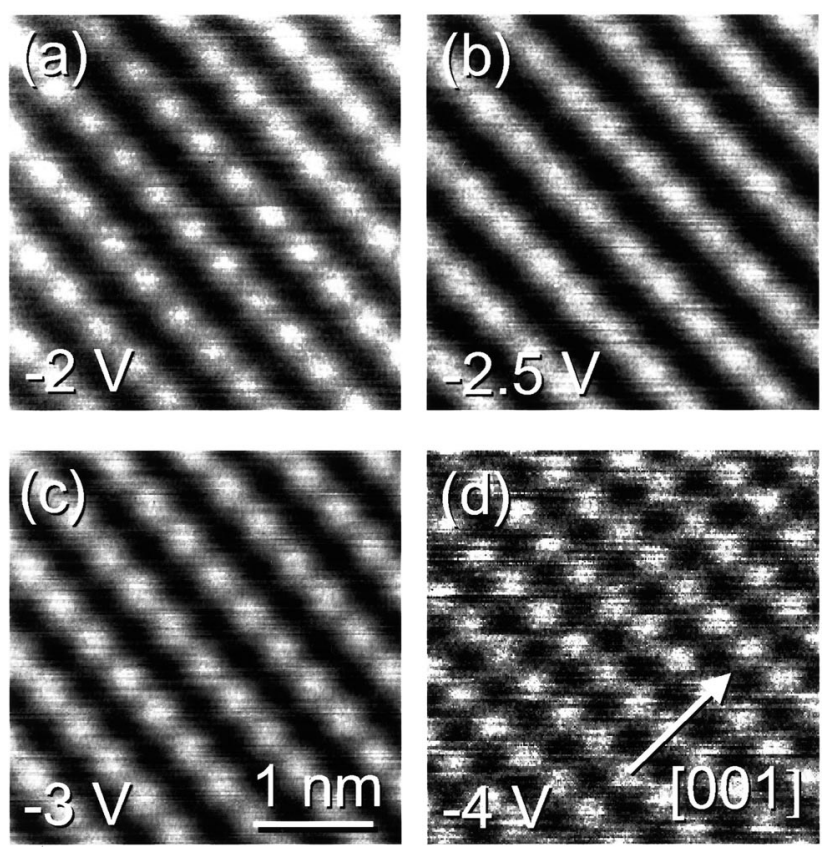

FIG. 1. Constant-current scanning tunneling microscopy images of a semi-insulating $\mathrm{GaAs}(110)$ surface acquired at (a) $-2.0 \mathrm{~V}$, (b) $-2.5 \mathrm{~V},(\mathrm{c})-3.0 \mathrm{~V}$, and (d) $-4.0 \mathrm{~V}$ tunneling voltage applied to the sample.

images match that of the highly doped, clean GaAs(110) surface. $^{12}$ We achieved stable atomically resolved imaging conditions for all sample voltages ranging from about -2 to $-6 \mathrm{~V}$. At smaller negative voltages the tunneling current became unstable and we observed that the tip touches and partly destroys the surface. No images could be obtained at positive sample voltages without photo carriers induced by illumination. Note that the STM images obtained at negative voltages predominantly exhibit rows along the [1 $\overline{1} 0]$ direction, i.e., the corrugation along the [001] direction is stronger

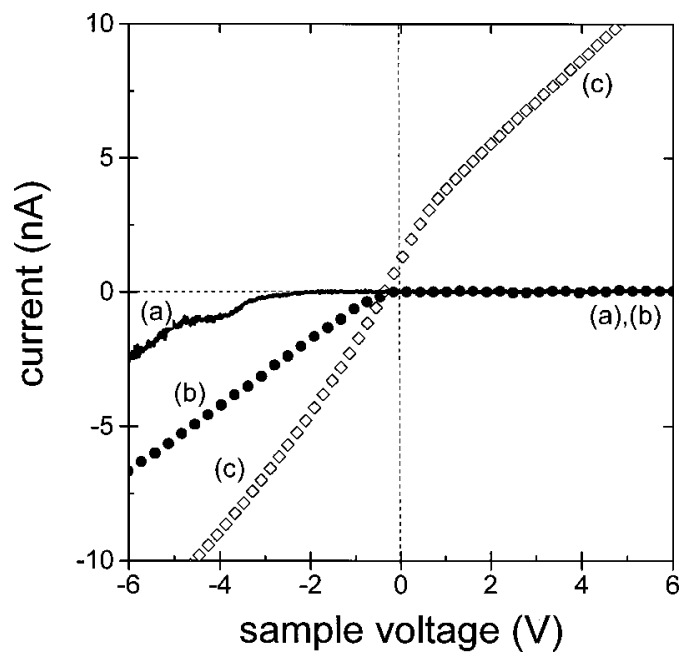

FIG. 2. Current-voltage spectra measured on semi-insulating GaAs(110) surfaces (a) in the dark in the tunneling mode, (b) in the dark in the point contact mode, and (c) under illumination with white light in the point contact mode.

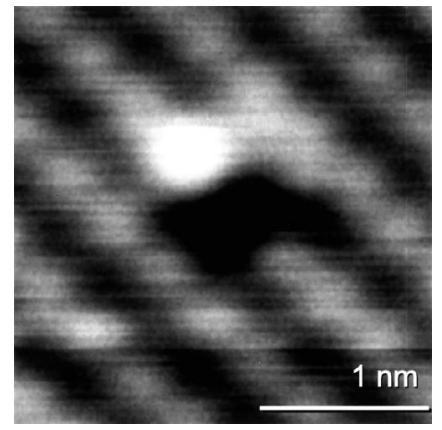

FIG. 3. Constant-current scanning tunneling microscopy image of a point defect identified as Schottky defect on a semi-insulating $\mathrm{GaAs}(110)$ surface acquired at $-3.0 \mathrm{~V}$ tunneling voltage applied to the sample.

than that along the $[1 \overline{1} 0]$ direction.

Figure 2 shows typical current-voltage characteristics of the cleaved semi-insulating GaAs(110) surface in the tunneling mode without illumination (spectrum a) and for increased sensitivity in the point contact mode with (spectrum c) and without (spectrum b) illumination with white light. In the dark (with no illumination) the tunneling (a) as well as the point contact (b) spectra only reveal current flow at negative voltages. No current could be extracted from the sample at positive voltages. This correlates with the observation that we did neither achieve any STM images under that condition. In contrast, light illumination results in a higher current at negative voltages and, unlike the dark case, a clear and strong current at positive voltages.

Figure 3 shows a constant-current scanning tunneling microscope image of a typical point defect, which we observed on the semi-insulating GaAs cleavage surfaces. The defect gives rise in the occupied density of states images to a local depression with dimensions of one dangling bond. The missing dangling bond indicates a vacancy-related defect. The defect does not exhibit any long-range height change around it. This indicates the absence of a local band bending induced by a charge. Thus these defects are uncharged on semiinsulating GaAs cleavage surfaces.

\section{DISCUSSION}

Figure 1 shows clearly that atomic resolution can be obtained on semi-insulating GaAs even at room temperature and in the dark. Previous works on materials with low conductivities only obtained atomic resolution at high temperatures, where the charge carriers are thermally excited. ${ }^{9}$ It is also important for further understanding to note that the socalled "low conductivity" in that and many other cases is more than four orders of magnitude higher than the conductivity of our semi-insulating GaAs samples. Furthermore, real insulating materials could so far only be imaged by STM as ultrathin films, where a tunneling through the insulating layer into the conducting substrate is possible. ${ }^{13}$ On semiinsulating $\mathrm{GaAs}$ no atomic resolution has been obtained previously and tunneling images were only acquired with photocarrier excitation by illumination. ${ }^{10}$ The comparison with previous works demonstrates that our results are obtained 
under different conditions, where the imaging process and the physical background of the local density of states imaged in the atomically resolved STM images is not clear at present. Therefore, we discuss in the following the experimental results by performing model calculations. First we concentrate on the tunneling mode, where the tip and the sample are separated by a vacuum barrier. Second, we discuss the point contact mode, i.e., the case where the tip touches the surface. On the basis of the experimental results and their comparison with the calculations, we extract the physics involved in measuring scanning tunneling microscopy images and spectra on semi-insulating GaAs.

\section{A. Tunneling through a vacuum barrier}

First of all we have to address the question of whether the STM images in Figs. 1 and 3 are indeed obtained in the tunneling mode, i.e., that the tunnel current flows through a vacuum barrier between the tip and the GaAs surface. The occasional occurrence of point defects as shown in Fig. 3 corroborates that all the images were obtained in the tunneling mode. If the STM images were obtained in the point contact mode, the surface would be destroyed (as we observed it indeed at positive voltages). At best one can only expect to observe a periodic pattern such as that found in atomic force microscopy (AFM) in the contact mode. Individual point defects could only be imaged by AFM in the noncontact mode ${ }^{14}$ where the tip is not touching the surface. Thus we obtained the STM images indeed in a noncontact mode, i.e., by tunneling through a vacuum barrier.

We now discuss the measurements performed in tunneling mode and with no illumination of the samples. The current vs voltage spectra in Fig. 2 show that in the dark (i) no current flows at positive voltages and (ii) current can only be extracted from the sample at negative sample voltages. In comparison, STM images were only obtained at negative voltages. These observations have important consequences:

(i) If no electrons can flow from the tip to the sample at positive voltages, the tip's occupied states cannot be facing, in terms of energy, the empty states of the GaAs(110) surface. Thus the tip-induced band bending must be so large that the tip's Fermi energy is below the conduction band edge at the GaAs surface. In this case the filled states of the tip never face the empty states of the GaAs surface energetically lying above the conduction band edge [see schematic drawing in Fig. 4(a)]. This situation is only possible if no carrier inversion is reached close to the surface, because with carrier inversion the band bending is reduced to approximately 0.7 $\mathrm{eV}$ as shown schematically in Fig. 4(b). Thus the lack of tunnel current infers the absence of a carrier inversion.

(ii) In comparison, if electrons can flow at negative voltages from the sample to the tip, one can conclude that the occupied states of the sample face the empty states of the tip. This is for sure the case when the valence band maximum of the GaAs sample is above the Fermi-energy of the tip. Thus, the fact that no stable current in the tunneling mode could be extracted for negative voltages smaller than $-2 \mathrm{~V}$ suggests that the valence band edge is approximately equal to the tip Fermi level close to $-2 \mathrm{~V}$. For negative voltages larger than
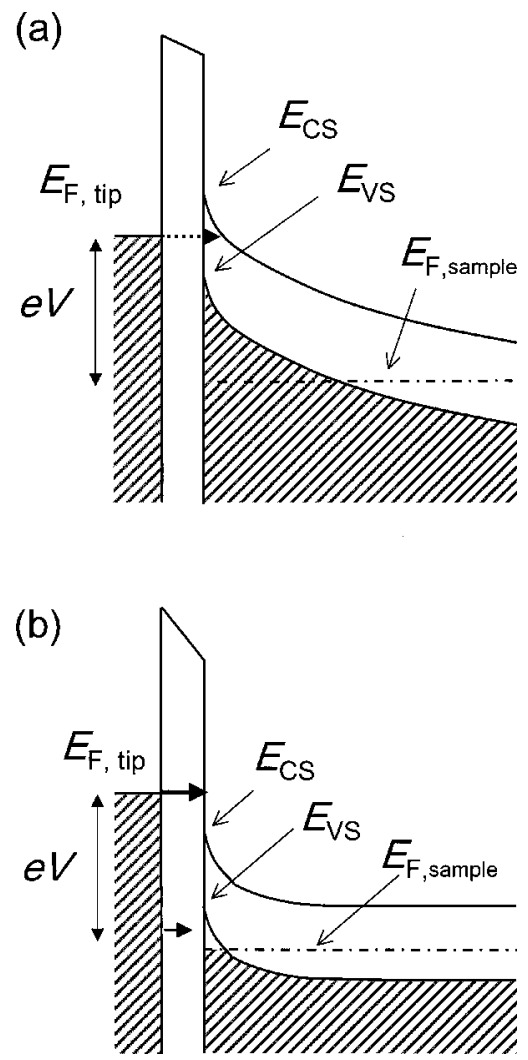

FIG. 4. Schematic drawing of the electron potential variation at a metal-vacuum-semi-insulating semiconductor interface (a) without inversion and (b) with inversion at positive voltages (eV) applied to the semiconductor. Note that the schematic is not to scale and the depletion width is much wider than the vacuum barrier. $E_{\mathrm{CS}}$ and $E_{\mathrm{VS}}$ are the energetic positions of the conduction and valence band edges at the surface, respectively. $E_{\mathrm{F} \text {,tip }}$ and $E_{\mathrm{F} \text {, sample }}$ are the Fermi energies of the tip and sample, respectively.

$-2 \mathrm{~V}(\mathrm{e} . \mathrm{g} .,-3 \mathrm{~V})$ the valence band edge must thus be above the tip Fermi level, since tunneling is possible.

These so far purely experimental conclusions can be corroborated and extended by calculations of the tip-induced band bending. ${ }^{15,16}$ In general, a difference between the tip and sample work functions causes an electric field between the two electrodes. This field is attenuated (screened) at the tip surface by the free electron gas of the metallic tip. In the semiconductor the field is screened by the net charge of free carriers (electrons and holes), ionized surface states, and ionized dopants. In the absence of sufficient surface states with energetic positions within the bulk band gap, the field penetrates into the semiconductor bulk. Thus the magnitude and extend of band bending in the semiconductor is governed by the difference in work functions of the tip and semiconductor, the applied voltage, the tip-sample distance, and the doping (and defect) concentration. It can be calculated by an integration of Poisson's equation. We followed the procedure described by Feenstra and Stroscio ${ }^{15}$ and Seiwatz and Green ${ }^{17}$ and calculated the positions of the conduction band $\left(E_{\mathrm{CS}}\right)$ and valence band edges $\left(E_{\mathrm{VS}}\right)$ at the surface shown in Fig. 5 for semi-insulating GaAs. We assumed a work function of $4.5 \mathrm{eV}$ for the metallic tip and a tip-sample separation 


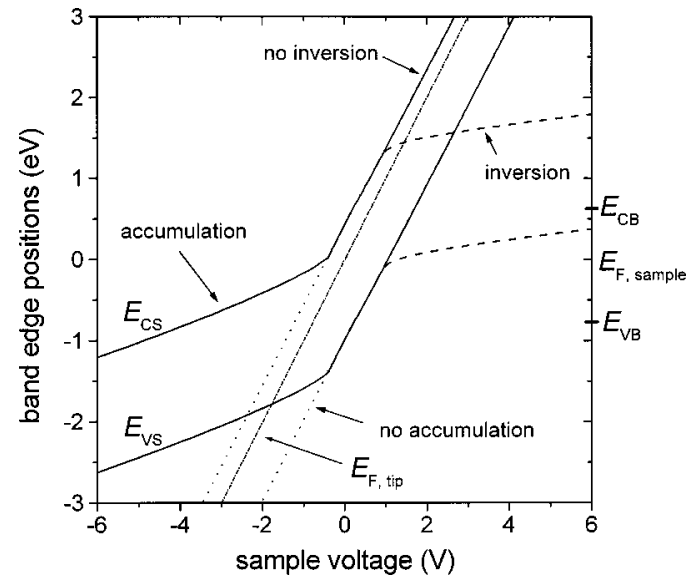

FIG. 5. Calculation of the energetic positions of the conduction band $E_{\mathrm{CS}}$ and valence band edges $E_{\mathrm{VS}}$ at the surface for a metalvacuum-semi-insulating GaAs system as a function of applied sample voltage. The energies are given relative to the bulk Fermi level of the semi-insulating GaAs $(0.768 \mathrm{eV}$ above the bulk valence band edge $E_{\mathrm{VB}}$, see right axis). Three cases are shown: The first case assumes that holes can gather in a surface inversion layer (dashed lines). The second case assumes that no accumulation zone exists (dotted lines). The solid line shows the case with accumulation but no carrier inversion. The dash-dotted diagonal line denotes the Fermi-energy position of the tip. A higher metal work function of the tip slightly shifts the curves to the left, but there are no qualitative changes.

of $0.9 \mathrm{~nm}$ as in Refs. 15 and 16. Furthermore, we used Fermi-Dirac statistics to calculate the position of the Fermienergy in the bulk from the concentration of EL2 defects $\left(1.3 \times 10^{16} \mathrm{~cm}^{-3}\right.$ deep donors), their defect level of $0.75 \mathrm{eV}$ below the conduction band, ${ }^{18}$ and a carbon acceptor concentration of $4.8 \times 10^{14} \mathrm{~cm}^{-3}$. The respective positions of the valence and conduction band edges in the bulk are indicated by dashes marked $E_{\mathrm{VB}}$ and $E_{\mathrm{CB}}$ on the right axis. Although the calculation of the band bending is based on a onedimensional model, ${ }^{19,20}$ we can discuss the main features related to the experimental data. We calculated in Fig. 5 three cases: (a) band bending with no carrier inversion at positive voltages but including accumulation at negative voltages (solid lines in Fig. 5), (b) band bending with inversion for positive voltages (dashed lines merging with solid lines), and (c) band bending with no accumulation at negative voltages (dotted lines merging with solid lines). The formalism of Ref. 17 also yields the electric field at the semiconductor surface. With this value we iteratively obtained the band edge positions inside the semiconductor for the three cases (Fig. 6). Three voltage ranges can be distinguished:

(i) For sample voltages between approximately -0.4 and $+0.7 \mathrm{~V}$ the positions of the band edges at the surface are practically identical for the three cases. The rise of the band bending is nearly directly proportional to the applied voltage. ${ }^{21}$ In this case the electric field is only screened by the charge of ionized donors. In this voltage range no tunneling of electrons from the STM tip or from the semiconductor sample is possible, because the conduction band edge at the surface is above the tip's Fermi level for positive

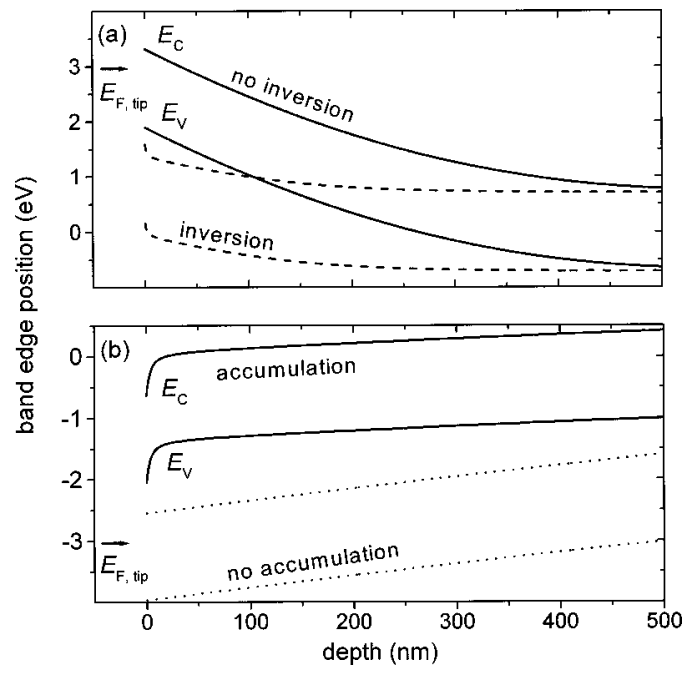

FIG. 6. Depth dependence of the position of the valence $\left(E_{\mathrm{V}}\right)$ and conduction $\left(E_{\mathrm{C}}\right)$ band edges for [(a) solid lines] positive voltages of $+3 \mathrm{~V}$ and no inversion, [(a) dashed lines] positive voltages of $+3 \mathrm{~V}$ and carrier inversion near the surface, [(b), solid lines] negative voltages of $-3 \mathrm{~V}$ and an accumulation of charge carriers at the surface, and [(b) dotted lines] negative voltages of $-3 \mathrm{~V}$ with no accumulation at the surface. The Fermi level of the tip is indicated by arrows. The Fermi level of the semi-insulating GaAs bulk is at $0 \mathrm{eV}$. Note that with slightly changing voltages the qualitative features do not change.

sample voltage, and the valence-band maximum at the surface is below the Fermi level of the tip for negative sample voltage.

(ii) For positive sample voltages greater than about $+1 \mathrm{~V}$ the valence band edge at the semiconductor surface is above the Fermi level in the semiconductor bulk (in Fig. 5 the valence band edge is above $0 \mathrm{eV}$, which is the position of the bulk Fermi level). In that case free holes are gathered in equilibrium conditions at the surface and the charge carrier inversion is formed. Due to the high density of states in the valence band, these holes screen most of the field within a few nanometers [see dashed lines in Fig. 6(a)], and thereby reduce the dependence of the band bending on the applied voltage (dashed lines in Fig. 5). With inversion the Fermi level of the tip is raised above the conduction band edge at the semiconductor surface and tunneling into empty surface states would be possible. However, carrier inversion occurs at the surface in equilibrium conditions. It can only form if free carriers are present in the semiconductor bulk valence band and if these carriers can reach the surface. Under tunneling conditions empty states in the top of the valence band are filled much faster than new holes can reach the surface in semi-insulating GaAs. Furthermore, the energy required for the thermal excitation of electrons into the conduction band is also too large to create significant charge carrier concentrations at the surface. Similarly, a tunneling of surface valence electrons into bulk conduction states through the barrier formed by the band bending is negligible, because the extent of the band bending into the bulk (about $300 \mathrm{~nm}$ ) makes the barrier too wide [see Fig. 6(a) solid lines]. Therefore, no inversion can be expected. If no free holes exist in 
the near surface region, the field continues to be solely screened by the charge of ionized donors and thus the band edges at the surface continue to rise at a rate nearly directly proportional with the applied voltage (as shown by the solid lines at positive voltages in Fig. 5). Consequently, for positive voltages the position of the conduction band edge at the surface is always above the Fermi energy of the tip (compare dashed-dotted line with solid lines in Fig. 5). Hence no filled tip states face empty sample states at the surface and as a consequence no current at positive voltages can be injected into surface states of semi-insulating GaAs. In addition, tunneling through the space charge region is also suppressed by the wide barrier an electron would have to overcome. In summary no tunneling is possible for positive sample voltages in agreement with the conclusions from our experimental observations (see Fig. 2), due to the absence of a charge carrier inversion and the absence of equilibrium conditions under tunneling conditions.

(iii) For sample voltages $V_{s}<-0.4 \mathrm{~V}$, the conduction band edge at the semiconductor surface is below the Fermi level in the semiconductor bulk. In equilibrium conditions, free electrons accumulate at the surface, effectively screening the field [Fig. 6(b), solid line] and reducing the slope of the solid curves in Fig. 5. In contrast, assuming no accumulation at the surface, only ionized acceptors can screen the field and the energetic positions of the band edges at the surface are nearly proportional to the applied voltage [dotted line in Figs. 5 and 6(b)]. In such a case no tunneling current can flow on the basis of the same arguments as in the previous paragraph.

In the experiment we, however, can extract a current. This is compatible with the case of accumulation, where the valence band edge at the surface is above the tip Fermi level for $V_{s}<-2 \mathrm{~V}$ (Fig. 5). This results in the onset of tunneling near $-2 \mathrm{~V}$ as seen in the tunneling spectra and in the STM images. Finally, the observation of mostly atomic rows along the [1 $1 \overline{1} 0]$ direction is indicative of the corrugation being dominated by filled-arsenic-derived dangling bond states. ${ }^{22,23}$ Note that in the nonequilibrium tunneling contact the contribution of the tunneling current from the accumulation layer near the surface is limited by the low conductivity in semiinsulating GaAs and thus is lower than the current from the valence band. Thus, the maxima in Fig. 1 correspond to the filled-arsenic-derived dangling bond states and not galliumderived surface states.

\section{B. Point contact}

We now focus on the case where the tip is in point contact with the semi-insulating GaAs. Without illumination we still observe no current at positive, but an increased current for negative, sample voltage. Based solely on the disappeared vacuum barrier, one would expect the current to be increased by four to ten orders of magnitude assuming a tip-sample separation of $0.4-1 \mathrm{~nm}$ in tunneling condition, due to the exponential distance dependence of the tunnel current. Such an increase in current, however, is not observed, indicating an additional physical effect reducing the tunnel current.

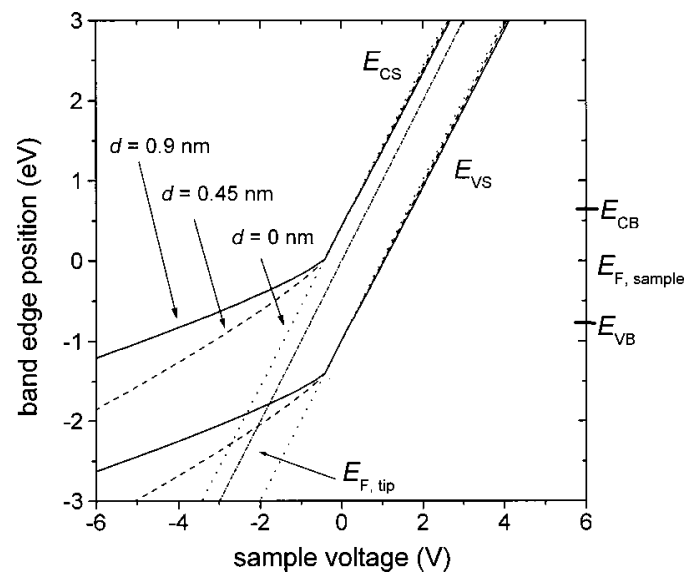

FIG. 7. Calculation of the energetic positions of the conduction band $E_{\mathrm{CS}}$ and valence band edges $E_{\mathrm{VS}}$ at the surface for a metalvacuum-semi-insulating GaAs system as a function of applied sample voltage for different tip-sample distances. The energies are given relative to the bulk Fermi level of the semi-insulating GaAs ( $0.768 \mathrm{eV}$ above the bulk valence band edge $E_{\mathrm{VB}}$, see right axis) and were calculated for the case of accumulation but no inversion at the surface. Note that the band bending at the surface increases with decreasing tip-sample separation.

In order to identify this physical effect, we calculated the band bending as a function of tip-sample separation. We assumed that no inversion takes place. Figure 7 shows the energetic positions of the conduction and valence band edges at the surface for $0.9,0.45$, and $0 \mathrm{~nm}$ tip-sample separation. In Fig. 8(a) and 8(b) the corresponding energetic positions are shown as a function of the distance from the surface into the bulk for sample voltages of +3 and $-3 \mathrm{~V}$, respectively. The following conclusions can be drawn.

(i) For positive voltages the band bending at the surface as well as into the bulk shows only a weak dependence on the tip-sample separation. Therefore, the same arguments of the tunneling case apply: Since no inversion occurs at the surface, the space charge region extends deeply into the bulk [Fig. 8(a)], such that tunneling through the space charge is negligible. Thus at positive sample voltages no current can be observed.

(ii) For negative voltages the situation is significantly different. The band bending increases strongly with decreasing tip-sample separation. In point contact the conduction and valence band edge at the surface are entirely defined by the difference of the work functions of sample and tip. Therefore, at the semiconductor surface the conduction band edge is $0.5 \mathrm{eV}$ above and the valence band edge is $1 \mathrm{eV}$ below the Fermi level of the tip (compare dotted lines with dasheddotted lines in Fig. 7). Thus, no current can flow from the semiconductor surface states into empty tip states. If any current flows, it must arise from tunneling through the space charge zone, which extends into the semiconductor bulk [see Fig. 8(b)]. A comparison of the depth dependence of the band edges shown in Fig. 8 already indicates that the barrier at negative sample voltages is much narrower than that at positive voltages, such that the tunnel current through the space charge region is not entirely negligible. In order to estimate this current contribution, we calculated the trans- 


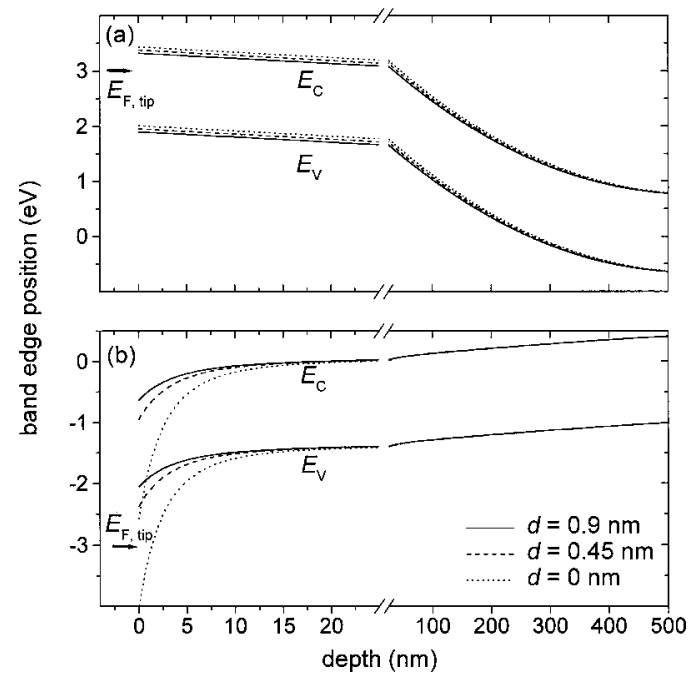

FIG. 8. Depth dependence of the position of the valence $\left(E_{\mathrm{V}}\right)$ and conduction $\left(E_{\mathrm{C}}\right)$ band edges for $($ a) positive voltages of $+3 \mathrm{~V}$ and no inversion and (b) negative voltages of $-3 \mathrm{~V}$ and an accumulation of charge carriers at the surface for three different tipsample separations. The Fermi level of the tip is indicated by arrows. The Fermi level of the semi-insulating GaAs bulk is at $0 \mathrm{eV}$. Note that with slightly changing voltages the qualitative features do not change.

mission coefficient through the space charge region as a function of electron energy using the WKB approximation. ${ }^{15}$ Figure 9 shows the result for three tip-sample distances at $-3 \mathrm{~V}$ sample voltage. The transmission coefficients through the vacuum barrier (at nonzero tip-sample distances) is shown as dashed lines, the transmission coefficients through the space charge region is shown as dotted lines and the total

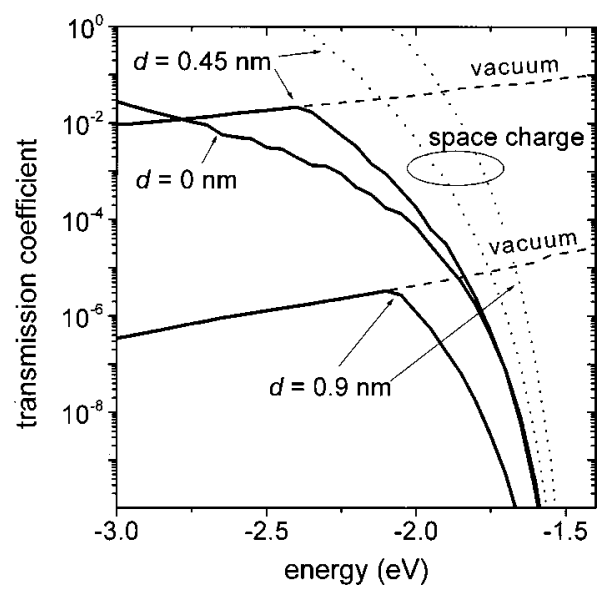

FIG. 9. Transmission coefficient as a function of electron energy for three different tip-sample separations for a metal-vacuumsemi-insulating GaAs system with $-3 \mathrm{~V}$ applied to the semiinsulating GaAs crystal. The dashed lines show the transmission coefficient only through the vacuum barrier between the sample and the tip. The dotted lines show the transmission coefficient only through the space charge region of the semi-insulating GaAs. The solid lines show the total transmission coefficient. Note that in the point contact the current is only limited by the tunneling of electrons through the space charge region.

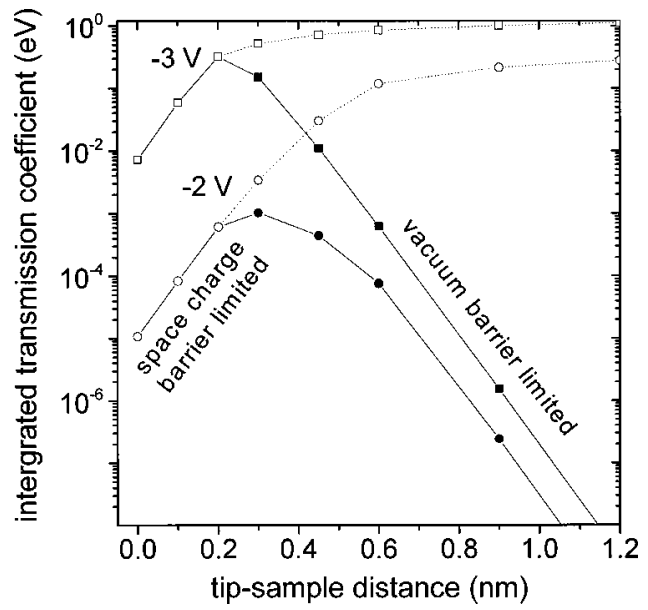

FIG. 10. Integrated transmission coefficient for sample voltages of -3 and $-2 \mathrm{~V}$ applied to the sample as a function of the tipsample separation for semi-insulating GaAs. At small tip-sample separation the transmission coefficient is limited by tunneling through the space charge barrier, whereas at larger tip-sample separation the limitation rises from the tunneling through the vacuum barrier between the tip and sample.

transmission coefficients are shown as solid lines. Figure 9 shows that with decreasing tip-sample distance the transmission through the vacuum barrier increases exponentially, while the transmission through the space charge region decreases. As a consequence, one can expect a maximum transmission current at some intermediate tip-sample distance, but not in the point contact. Figure 10 shows the transmission coefficients integrated from the Fermi level of the tip up to the top of the valence band in the semiconductor bulk as a function of the tip-sample separation. The open symbols represent the integrated transmission coefficient through the space charge region only, whereas the filled symbols show the transmission coefficient for tunneling through the vacuum barrier. The solid line indicates the combined transmission coefficient trough space charge region and vacuum barrier. For small tip-sample separations the valence-band current is limited by tunneling through the space charge region, whereas for large separation the vacuum barrier limits the current. The result in Fig. 10 also shows that one cannot expect that the current increases by four to ten orders of magnitude solely by reducing the tip-sample distance from about $0.4-1 \mathrm{~nm}$ down to zero distance (point contact). One rather can only expect about $1 \pm 1$ order of magnitude current increase. This is in agreement with our observation in Fig. 2.

\section{Tunneling under illumination}

Illumination with white light excites electrons from the valence band into the conduction band in the near-surface region. Although this effect increases the charge carrier concentration in the near surface region, the band bending at the surface is still controlled by the difference in work function between the tip and sample materials. The increase in free charge carriers (with carrier inversion) only increases the screening and thus decreases the extent of the band bending 
into the semiconductor bulk. This extent of the band bending into the bulk is limiting the transmission, such that in the dark current can only flow at negative voltages. With illumination the screening is enhanced and tunneling through the space charge region becomes possible at negative and positive voltages. Note that illumination of the sample with white light increases the conductivity of the sample's surface only, but not in the bulk. Thus the current observed now is still limited by the carrier transport through the sample.

\section{Point defects on semi-insulating GaAs surfaces}

We observed only uncharged point defects on the (110) cleavage surfaces of semi-insulating GaAs. This is in contrast to the observation of a rich variety of charged point defects and dopant atoms in cleavage surfaces of highly doped III-V and II-VI semiconductors. ${ }^{24}$ In order to discuss the origin of such defects, we first identify the defects.

Each defect appears as one missing dangling bond, which indicates a missing As atom. In addition, one of the neighboring dangling bonds is raised. Such a signature can have several origins: (i) A dopant-vacancy complex has a similar morphology, ${ }^{25}$ but the concentration of carbon dopant atoms is much too low to explain the concentration of defects. Thus the defect must be a native defect. (ii) An isolated uncharged anion vacancy has a symmetric structure. ${ }^{26}$ Therefore the defect cannot be an isolated As vacancy. (iii) The observed signature is, however, typical for a surface anion vacancycation vacancy pair (Schottky defect). Such a defect gives rise to one brighter dangling bond neighboring to one missing dangling bond. Furthermore, it is conceivable that the anion-cation vacancy pair is electrically uncharged, because the anions and cations are removed stoichiometrically. Schottky defects were indeed observed previously on (110) cleavage surfaces of InSb. ${ }^{27}$

If a defect would be charged on the semi-insulating GaAs surface, it would imply the presence of a charge carrier. However, the concentration of thermally excited carriers or of dopant atoms is much too low to accommodate for the defect concentration on the surface. Thus for semi-insulating GaAs uncharged defects are more stable than charged defects, due to the lack of free charge carriers. This is consistent with the observation of uncharged Schottky defects.

\section{CONCLUSIONS}

In summary, we demonstrated that stable atomic resolution in scanning tunneling microscopy images can be achieved on (110) cleavage surfaces of semi-insulating GaAs with resistivities as high as $1.2 \times 10^{7} \Omega \mathrm{cm}$, i.e., more than four orders of magnitude higher than previously achieved. The experiments showed that at room temperature and in the dark, only the occupied dangling bond states localized at the arsenic surface atoms can be imaged. No tunnel current can be extracted at positive sample voltages without photoexcitation of carriers. From these experimental results we extracted, with help of simple model calculations, the physical mechanisms that allow the extraction of stable current in STM configurations. At positive sample voltages no carrier inversion occurs during scanning tunneling microscopy, because the carrier system does not reach equilibrium under tunneling conditions. As a consequence no STM is possible at positive voltages without photocarrier excitation. At negative sample voltages an accumulation layer is formed and sufficiently maintained under tunneling conditions. In the tunneling mode through a vacuum barrier the band bending then remains small enough such that tunneling from the occupied valence band of the GaAs surface into the empty tip states is possible. In the point contact mode the band bending still remains too large, such that no filled GaAs surface states face empty tip states. Current flow is only maintained by tunneling through the space charge region. With illumination with white light and subsequent photocarrier excitation, tunneling through the space charge region in the point contact is enhanced due to the increased screening and thus smaller space charge barrier. The results show that as long as the absolute resistance through the sample is still smaller than the tunneling resistance of the vacuum barrier, semiinsulating materials can be imaged including those found in electronic devices, without the need to excite carriers at high temperatures or with light. Furthermore, the results show that other low-conductivity materials without surface states in the band gap can also be investigated by STM without carrier excitation, if the occupied states are imaged and the accumulation layer is sufficiently maintained under tunneling condition, such that the band bending is not too large. The limit of this tunneling process depends on the material's properties, notably the size of the band gap and the remaining (unintentional) impurity doping and defect concentrations.

\section{ACKNOWLEDGMENTS}

The authors thank Dr. Jurisch from Freiberger Compound Materials for the excellent sample analysis, Dr. Toporowski for providing the samples, the Deutsche Forschungsgemeinschaft as well as the Director, Office of Energy Research, Office of Basic Energy Research, Materials Science Division, U.S. Department of Energy under Contract No. DEAC03-76SF00098 for financial support.
${ }^{1}$ M. Pfister, M. B. Johnson, S. F. Alvarado, H. W. M. Salemink, U. Marti, D. Martin, F. Morier-Genoud, and F. K. Reinhart, Appl. Phys. Lett. 65, 1168 (1994).

${ }^{2}$ R. M. Feenstra, Semicond. Sci. Technol. 9, 2157 (1994).

${ }^{3}$ N. Liu, J. Tersoff, O. Baklenov, A. L. Holmes, Jr., and C. K. Shih, Phys. Rev. Lett. 84, 334 (2000).
${ }^{4}$ S. Gwo, K. J. Chao, C. K. Shih, K. Sadra, and B. G. Streetman, Phys. Rev. Lett. 71, 1883 (1993).

${ }^{5}$ A. Y. Lew, E. T. Yu, D. H. Chow, and R. H. Miles, Appl. Phys. Lett. 65, 201 (1994).

${ }^{6}$ J. Steinshnider, J. Harper, M. Weimer, C.-H. Lin, S. S. Pei, and D. H. Chow, Phys. Rev. Lett. 85, 4562 (2000). 
${ }^{7}$ R. S. Goldman, R. M. Feenstra, B. G. Briner, M. L. O'Steen, and R. J. Hauenstein, Appl. Phys. Lett. 69, 3698 (1996).

${ }^{8}$ J. F. Zheng, J. D. Walker, M. B. Salmeron, and E. R. Weber, Phys. Rev. Lett. 72, 2414 (1994).

${ }^{9}$ The samples investigated by M. R. Castell, C. Muggelberg, G. A. D. Briggs, and D. T. Goddard, J. Vac. Sci. Technol. B 14, 966 (1996) have a resistivity coming closest to our semi-insulating material (250 vs $10^{7} \Omega \mathrm{cm}$ for our samples). Even though no scanning tunneling microscopy investigation was possible without additional thermal carrier excitation. Note that all other STM investigations of metal oxides used highly conductive samples, whose properties were equivalent to carrier concentrations ranging from typically $10^{18}-10^{22} \mathrm{~cm}^{-3}$ : D. A. Bonnell, Prog. Surf. Sci. 57, 187 (1998), and references therein.

${ }^{10}$ G. F. A. van de Walle, H. van Kempen, P. Wyder, and P. Davidsson, Appl. Phys. Lett. 50, 22 (1987).

${ }^{11}$ M. Toporowski, Ph.D. thesis, RWTH Aachen, 1997.

${ }^{12}$ R. M. Feenstra, J. A. Stroscio, J. Tersoff, and A. P. Fein, Phys. Rev. Lett. 58, 1192 (1987).

${ }^{13}$ J. Viernow, D. Y. Petrovykh, A. Kirakosian, J.-L. Lin, F. K. Men, M. Henzler, and F. J. Himpsel, Phys. Rev. B 59, 10356 (1999).

${ }^{14}$ Y. Sugawara, M. Ohta, H. Ueyama, and S. Morita, Science 270, 1646 (1995).

${ }^{15}$ R. M. Feenstra and J. A. Stroscio, J. Vac. Sci. Technol. B 5, 923 (1987).
${ }^{16}$ R. Maboudian, K. Pond, V. Bressler-Hill, M. Wassermeier, P. M. Petroff, G. A. D. Briggs, and W. H. Weinberg, Surf. Sci. 275, L662 (1992).

${ }^{17}$ R. Seiwatz and M. Green, J. Appl. Phys. 29, 1034 (1958).

${ }^{18}$ C. H. Henry and D. V. Lang, Phys. Rev. B 15, 989 (1977).

${ }^{19}$ R. Dombrowski, C. Steinebach, C. Wittneven, M. Morgenstern, and R. Wiesendanger, Phys. Rev. B 59, 8043 (1999).

${ }^{20}$ M. Wenderoth, M. A. Rosentreter, K. J. Engel, A. J. Heinrich, M. A. Schneider, and R. G. Ulbrich, Europhys. Lett. 45, 579 (1999).

${ }^{21}$ M. Weimer, J. Kramar, and J. D. Baldeschwieler, Phys. Rev. B 39, 5572 (1989).

${ }^{22} \mathrm{Ph}$. Ebert, B. Engels, P. Richard, K. Schroeder, S. Blügel, C. Domke, M. Heinrich, and K. Urban, Phys. Rev. Lett. 77, 2997 (1996).

${ }^{23}$ N. D. Jäger, E. R. Weber, and M. Salmeron, J. Vac. Sci. Technol. B 19, 511 (2001).

${ }^{24} \mathrm{Ph}$. Ebert, Surf. Sci. Rep. 33, 121 (1999), and references therein.

${ }^{25} \mathrm{Ph}$. Ebert, M. Heinrich, M. Simon, C. Domke, K. Urban, C. K. Shih, M. B. Webb, and M. G. Lagally, Phys. Rev. B 53, 4580 (1996).

${ }^{26} \mathrm{Ph}$. Ebert, K. Urban, and M. G. Lagally, Phys. Rev. Lett. 72, 840 (1994).

${ }^{27}$ L. J. Whitman, J. A. Strocio, R. A. Dragoset, and R. J. Celotta, J. Vac. Sci. Technol. B 9, 770 (1991). 